\title{
Computations in quantum mechanics made easy
}

\author{
H J Korsch ${ }^{1}$ and K Rapedius ${ }^{2}$ \\ ${ }^{1}$ FB Physik, Universität Kaiserslautern, D-67653 Kaiserslautern, Germany \\ ${ }^{2}$ Karlsruhe Institute of Technology (KIT), Adenauerring 2, D-76131 Karlsruhe, \\ Germany \\ E-mail: korsch@physik.uni-kl.de, kevin.rapedius@kit.edu
}

\begin{abstract}
Convenient and simple numerical techniques for performing quantum computations based on matrix representations of Hilbert space operators are presented and illustrated by various examples. The applications include the calculations of spectral and dynamical properties for one-dimensional and two-dimensional singleparticle systems as well as bosonic many-particle and open quantum systems. Due to their technical simplicity these methods are well suited as a tool for teaching quantum mechanics to undergraduates and graduates. Explicit implementations of the presented numerical methods in MATLAB are given.
\end{abstract}

Submitted to: Eur. J. Phys.

\section{Introduction}

In [1] it was shown how to calculate the spectra of one-dimensional quantum systems in a simple, convenient and effective way by means of matrix representations of Hilbert space operators. Here we extend these techniques in various ways including the computation of dynamical properties as well applications to higher dimensional systems, bosonic many-particle and open quantum systems.

As discussed in [1] the basic building blocks for the discrete matrix representation of operators used in the following programs are the operators $\hat{a}$ and $\hat{a}^{\dagger}$, well known from the harmonic oscillator, where they act as ladder operators on the harmonic oscillator eigenstates $|n\rangle, n=0,1,2, \ldots$ :

$$
\hat{a}|n+1\rangle=\sqrt{n+1}|n\rangle, \hat{a}^{\dagger}|n\rangle=\sqrt{n+1}|n+1\rangle, \hat{a}^{\dagger} \hat{a}|n\rangle=n|n\rangle .
$$

Motivated, e.g., by the application to the radiation field described by harmonic oscillators with frequency $\omega_{0}$ these operators create or annihilate a photon of this frequency or, more generally, a bosonic particle in second quantization. Therefore these 
operators are also known as creation and annihilation operators. Here we will mainly use the matrix representation of these operators in the harmonic oscillator basis:

$$
\hat{a}=\left(\begin{array}{ccccc}
0 & \sqrt{1} & 0 & 0 & \ldots \\
0 & 0 & \sqrt{2} & 0 & \ldots \\
0 & 0 & 0 & \sqrt{3} & \ldots \\
0 & 0 & 0 & 0 & \ldots \\
\vdots & \vdots & \vdots & \vdots & \ddots
\end{array}\right) .
$$

As an introduction to the numerical applications presented below, the following short MATLAB code shows the construction of these matrices and their application to a basis vector

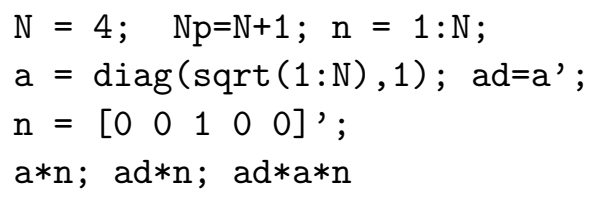

where the properties (1) can be tested. It should be noted, however, that such numerical matrix representations are necessarily finite, which causes numerical errors. The reader may try for example to test the validity of the commutation relation $\left[\hat{a}, \hat{a}^{\dagger}\right]=1$ numerically or to construct the eigenstates of the annihilation operator $\hat{a}$, the coherent states, numerically. In many cases these finite size errors can be reduced by increasing the matrix size.

In addition, we will use the representation of the position and momentum operators

$$
\hat{x}=\frac{s}{\sqrt{2}}\left(\hat{a}^{\dagger}+\hat{a}\right), \quad \hat{p}=\frac{\mathrm{i}}{s \sqrt{2}}\left(\hat{a}^{\dagger}-\hat{a}\right)
$$

where the scaling parameter is chosen as $s=1$ in the following (see [1] for details). These matrix representations of operators can now be used to construct in a simple way matrix representations of other operators, such as the Hamiltonian or time-evolution operators.

This paper is organized as follows: In section 2 we briefly review the calculation of quantum eigenvalues for one-dimensional systems [1] and extend the analysis to time-dependent calculations. The example applications include Bloch-oscillations in a tilted periodic lattice. Section 3 illustrates the use of matrix representation for angular momentum operators. In section 4 it is shown how to calculate the spectrum of a two-dimensional quantum system. Applications to bosonic many-particle systems are illustrated in section 5 by means of the Bose-Hubbard model. Finally, the dynamics of an open quantum system described by a Lindblad master equation is calculated in section 6 .

\section{One-dimensional systems}

As a first example we consider the calculation of bound state energies for a single particle in a one-dimensional potential described by the Hamiltonian

$$
\hat{H}=\frac{\hat{p}^{2}}{2 m}+V(\hat{x}),
$$


which has already been discussed in [1] for harmonic and quartic potentials. There a simple program code can be found for computing the eigenvalues and, in addition, also the corresponding wave functions. As an introduction we also list this code here, however for a simple double-well potential

$$
V(x)=\frac{1}{2}\left(|x|-x_{0}\right)^{2},
$$

a potential with harmonic minima at $\pm x_{0}$ separated by a barrier of height $x_{0}^{2} / 2$ at $x=0$. In the program code

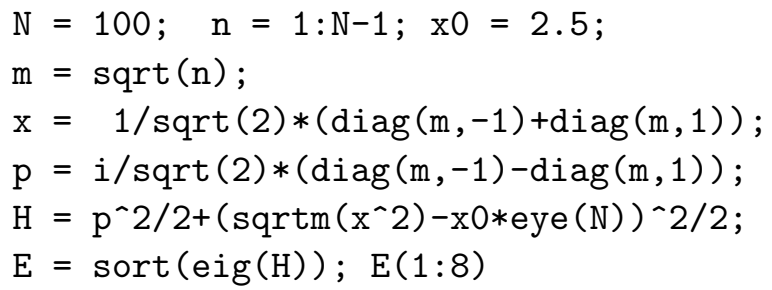

where $\hbar$ and the particle mass $m$ are chosen as unity and $x_{0}=2.5$. In addition it should be noted that the matrix function $|x|$ has been generated by taking the square $x^{\wedge} 2$ followed by the MATLAB matrix function $\operatorname{sqrtm}\left(x^{\wedge} 2\right)$. The output of the program is a listing of the first eight energy eigenvalues $E_{n}, n=0, \ldots, 7$, where the eigenvalues below the potential barrier are arranged in doublets. Let us have a brief look at the time dynamics for an initial state chosen as the ground state of the right potential well. This can be achieved by adding the lines

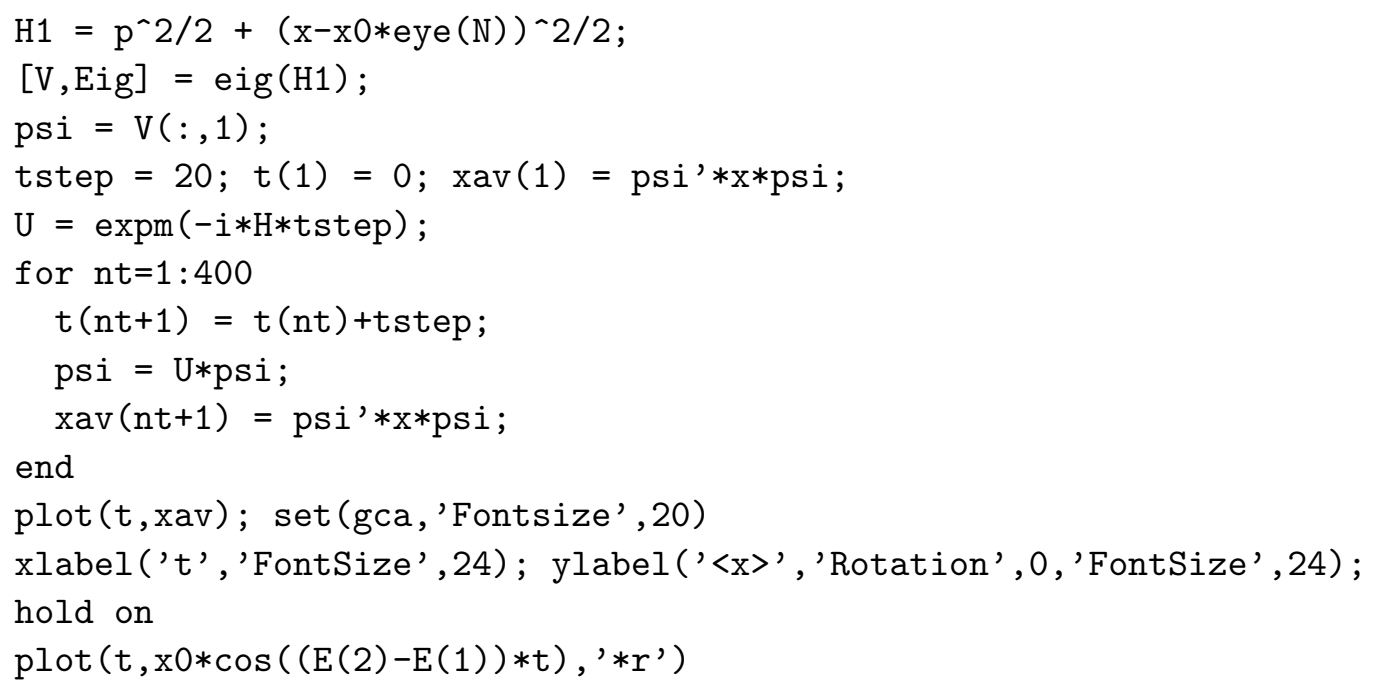

to the program above. Then the time evolution operator $\hat{U}=\exp -\mathrm{i} \hat{H} \Delta t / \hbar$ is used to propagate the wave function over a sequence of 80 time steps $\Delta t=20$. At each step the expectation value $\langle\hat{x}\rangle$ is computed and finally plotted as a function of time in comparison with the approximation $x(t)=x_{0} \cos \left(\left(E_{1}-E_{0}\right) t\right)$ as shown in figure 1 .

As a second application let us consider an extended one-dimensional system, a particle in a periodic potential $V_{0}(x+d)=V_{0}(x)$ accelerated by a constant force $F$ :

$$
V(x)=V_{0}(x)+F x
$$

In the tight-binding approximation, the Hamiltonian is expressed in terms of the Wannier states $|n\rangle$ of the lowest band of the periodic potential, which are localized 


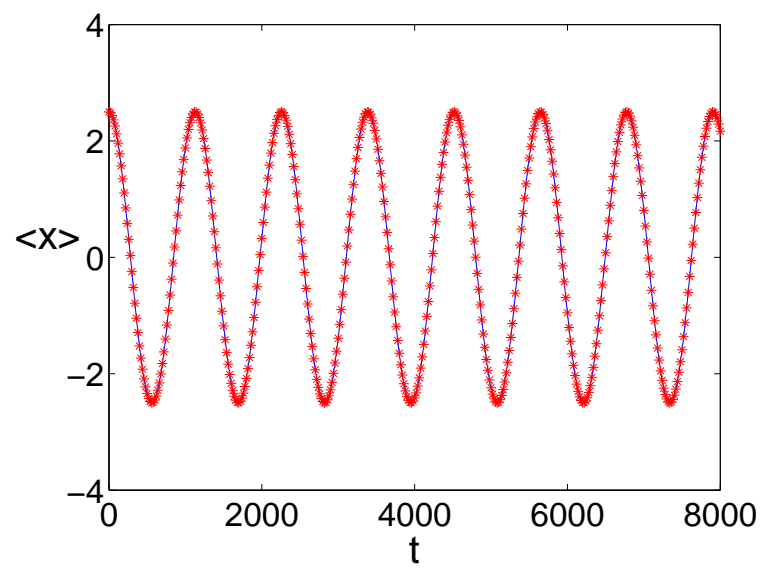

Figure 1. Double well potential: Expectation value $\langle\hat{x}\rangle$ as a function of time for a Gaussian wave packet initially at the right well (blue line) compared to the approximation $x(t)$ (red stars).

at the potential minima numbered by $n$. Taking only transitions between neighboring wells into account, the tight-binding Hamiltonian reads

$$
\hat{H}=\sum_{n}(\epsilon+d F n)|n\rangle\langle n|-\frac{\Delta}{4}(|n+1\rangle\langle n|+| n\rangle\langle n+1|)
$$

(see, e.g., [2] and references therein), where $\epsilon$ is the band energy and $\Delta$ is the bandwidth. For $F=0$ we have an almost free motion with quasimomentum $\kappa$ for energies inside the band $E(\kappa)=\epsilon+\frac{\delta}{2} \cos \kappa$, whereas for $F \neq 0$ the particle is, contrary to a naive expectation, confined to a finite region in space performing an oscillatory motion with a period $T_{B}=2 \pi \hbar / d F$, the so-called Bloch period. This is closely related to the fact that the Hamiltonian possesses the equidistant eigenvalues $E_{n}=\epsilon+d F n, n=0, \pm 1, \pm 2, \ldots$ This Bloch oscillation is illustrated by the following program for $F=0.005, d=2 \pi$, $\Delta=1, \hbar=1$ and an array extending from $n=-60$ to $n=60$.

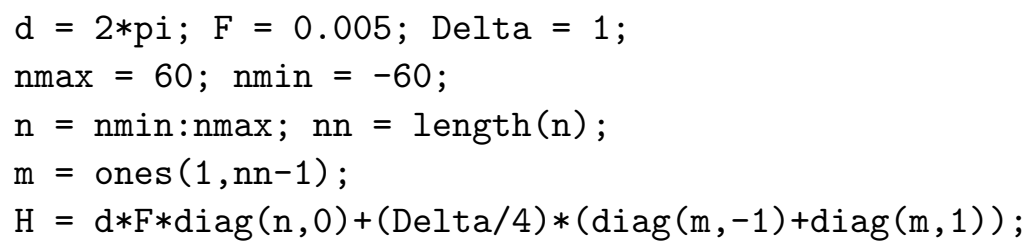

The matrix representation of the Hamiltonian (7) in the basis $|n\rangle$ is tridiagonal. Its construction is very similar to the one used for the $x$ matrix in the program above. Here, however, there are also non-vanishing entries along the diagonal and the other matrix elements are constant. The interested reader can easily calculate the eigenvalues of this Hamiltonian numerically and compare with the analytical result given above. This will show that the results agree with the exception of the regions close to the boundary of the finite $n$-array, where the numerical values are not yet converged. In the following we will study the time evolution of an initial wave packet. We first consider a wave packet, localized initially at site $n=0$, which is propagated over $N=2$ Bloch periods, each one is discretized by $J=80$ time steps: 

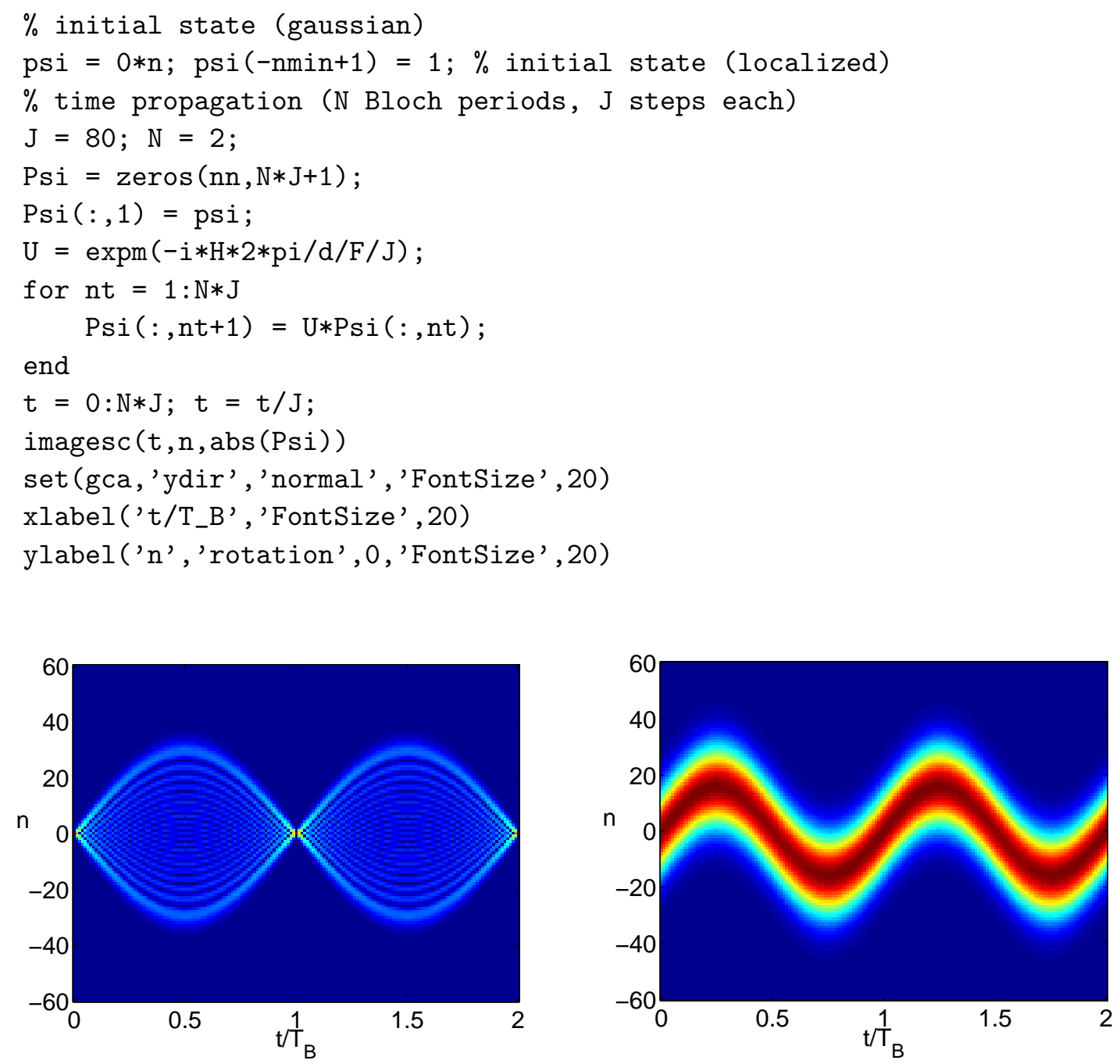

Figure 2. Bloch oscillation: Breathing (left) and oscillatory mode (right) for an initially narrow or extended distribution [2].

The resulting wave function $\psi_{n}(t)$ is plotted as a color map in the left panel of figure 2. This is a breathing mode, where the wave packet oscillates periodically in the region $|n|<\frac{\Delta}{d F}\left|\sin \pi t / T_{B}\right|$ (see [2] for more information). If a broad initial wave function is chosen, the dynamics changes. For example an initial Gaussian distribution, realized in the program by replacing the line marked as initial state (localized) by the lines

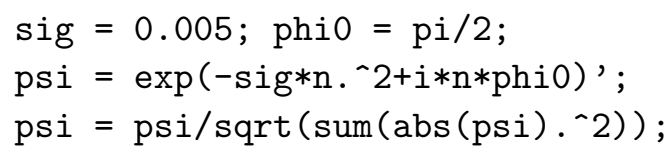

leads to an oscillating mode as shown in the right panel of figure 2 known as the Bloch oscillation.

Most remarkably, such a Bloch oscillation leads to an almost dispersionless directed transport during the first quarter of the Bloch period. Then the motion continues in the opposite direction, which suggests that we can prevent this backward motion by a flip of the field direction at this time. Continuing this process periodically, one can expect 
a directed dispersionless transport. To study such a field-flip system numerically, we first replace the second line in the program above by nmax $=160 ; \mathrm{nmin}=-40$; and the Hamiltonian $\mathrm{H}$ by the two lines

$$
\begin{aligned}
& \mathrm{Hp}=+\mathrm{d} * \mathrm{~F} * \operatorname{diag}(\mathrm{n}, 0)+(\operatorname{Delta} / 4) *(\operatorname{diag}(\mathrm{m},-1)+\operatorname{diag}(\mathrm{m}, 1)) ; \\
& \mathrm{Hm}=-\mathrm{d} * \mathrm{~F} * \operatorname{diag}(\mathrm{n}, 0)+(\operatorname{Delta} / 4) *(\operatorname{diag}(\mathrm{m},-1)+\operatorname{diag}(\mathrm{m}, 1)) ;
\end{aligned}
$$

defining Hamiltonians with different signs of $F$. As an initial state, a Gaussian is chosen and, for the time-propagation, the corresponding time-evolution operators are defined, which are then applied alternately during the subsequent Bloch periods:

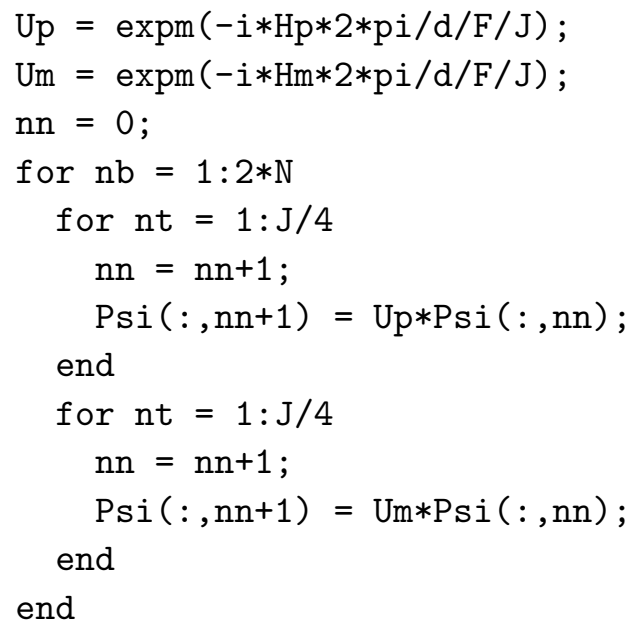

The resulting dynamics shown in figure 3 agrees with our conjecture. Most remarkably, however, one can show that the transport velocity is given by $v=\Delta d / \pi \hbar$, which is independent of the force $F[2]$.

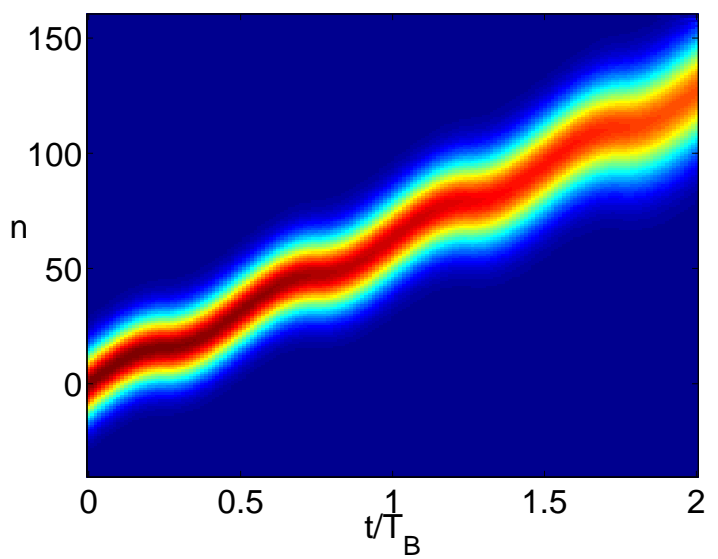

Figure 3. Bloch oscillation: Directed almost dispersionless transport for a field flipped periodically with twice the Bloch frequency.

\section{Angular momentum operators}

In a similar manner one can construct the matrix representation of the angular momentum operators $\hat{J}_{x}, \hat{J}_{y}, \hat{J}_{z}$ in the basis of eigenstates $|j, m\rangle$ of the operator $\hat{J}_{z}$ 
with eigenvalues $m=-j,-j+1, \ldots,+j$, where $j \geq 0$ is the total angular momentum, which is an even or odd multiple of $\frac{1}{2}$. First we construct the ladder operators $\hat{J}_{+}$and $\hat{J}_{-}=\hat{J}_{+}^{\dagger}$ with $\hat{J}_{+}|j, m\rangle=\sqrt{j(j+1)-m(m+1)}|j, m+1\rangle$ and the relations

$$
\hat{J}_{x}=\frac{1}{2}\left(\hat{J}_{-}+\hat{J}_{+}\right), \quad \hat{J}_{y}=\frac{i}{2}\left(\hat{J}_{-}-\hat{J}_{+}\right), \quad \hat{J}_{z}=\frac{1}{2}\left[\hat{J}_{+}, \hat{J}_{-}\right]
$$

coded in the following program lines for $j=2$ :

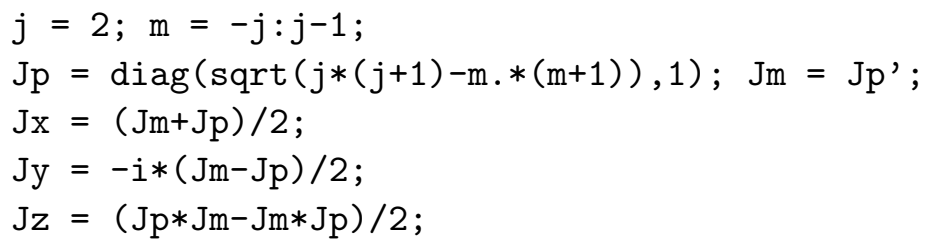

If desired, one can check here the angular momentum commutation relations $\left[\hat{J}_{x}, \hat{J}_{y}\right]=$ $\mathrm{i} \hat{J}_{z}$ by means of $\mathrm{Jx} * \mathrm{Jy}-\mathrm{Jy} * \mathrm{Jx}-i * \mathrm{Jz}$, which should yield the zero matrix. As an application, one can calculate the energy eigenvalues of the rigid body Hamiltonian $(\hbar=1)$

$$
\hat{H}=\frac{\hat{J}_{x}^{2}}{2 I_{x}}+\frac{\hat{J}_{y}^{2}}{2 I_{y}}+\frac{\hat{J}_{z}^{2}}{2 I_{z}},
$$

where the $I_{x}, I_{y}$ and $I_{z}$ are the principal moments of inertia. For a symmetric top, the eigenvalues of the Hamiltonian are well known, namely

$$
E_{j k}=\frac{j(j+1)}{2 I_{x}}+\left(\frac{1}{2 I_{z}}-\frac{1}{2 I_{x}}\right) k^{2}, \quad k=-j, \ldots, j
$$

for $I_{x}=I_{y} \neq I_{z}$. For an asymmetric top, however, the eigenvalues for the special cases $j=1,2,3$ are given in [3], but no general formula exists. This motivates, of course, a numerical approach, which is achieved by adding the program lines

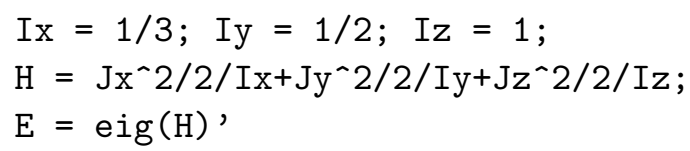

in order to calculate the energy eigenvalues of an asymmetric top with $I_{x}=1 / 3$, $I_{y}=1 / 2$ and $I_{z}=1$ for $j=2$. Note, however, that for this system body-fixed angular momenta must be used with commutation relations $\left[\hat{J}_{x}, \hat{J}_{y}\right]=-\mathrm{i} \hat{J}_{z}$ (see, e.g., 3, 4] for an explanation). This can be achieved by changing the sign of the matrix representing $\hat{J}_{y}$. This subtlety does not affect the eigenvalues of the Hamiltonian (9) because it depends only of the squares of the operators. The numerical eigenvalues

$$
\mathrm{E}=4.2679 \quad 4.5000 \quad 6.0000 \quad 7.5000 \quad 7.7321
$$

given by the program agree with the formulas in [3]:

$E_{1}=\frac{2}{I_{z}}+\frac{1}{2 I_{x}}+\frac{1}{2 I_{y}}, \quad E_{2}=\frac{2}{I_{y}}+\frac{1}{2 I_{z}}+\frac{1}{2 I_{x}}, \quad E_{3}=\frac{2}{I_{x}}+\frac{1}{2 I_{y}}+\frac{1}{2 I_{z}}$,

$E_{4,5}=\frac{1}{I_{x}}+\frac{1}{I_{y}}+\frac{1}{I_{z}} \pm \sqrt{\left(\frac{1}{I_{x}}+\frac{1}{I_{y}}+\frac{1}{I_{z}}\right)^{2}-3\left(\frac{1}{I_{x} I_{y}}+\frac{1}{I_{y} I_{z}}+\frac{1}{I_{z} I_{x}}\right)}$.

Such a small value as $j=2$ is, of course, not a challenge for a computational treatment. The interested reader may try, for instance, the larger angular momentum 


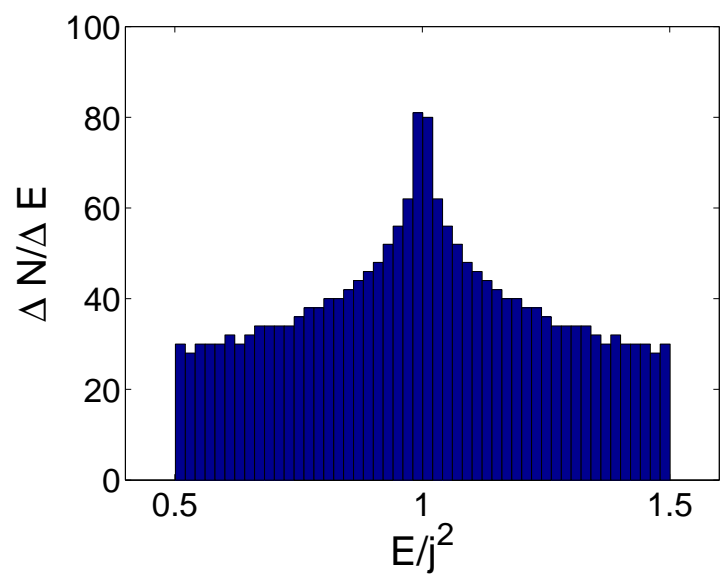

Figure 4. Asymmetric top: Histogram of the density of energy eigenvalues for rotational momentum $j=1000$ showing a pronounced spike at the classical energy at the saddle point of the energy surface.

$j=1000$ and analyze the distribution of the eigenvalues by plotting the state density via hist $\left(E / j^{\wedge} 2,50\right)$. The resulting distribution in figure 4 shows a clear restriction to an energy interval and a pronounced maximum. This structure can be understood by the observation that for large angular momentum the system behaves almost classically. Here the classical dynamics is restricted to a sphere with constant angular momentum $|\vec{J}|=j$ and the energy function $H=\frac{J_{x}^{2}}{2 I_{x}}+\frac{J_{y}^{2}}{2 I_{y}}+\frac{J_{z}^{2}}{2 I_{z}}$ possesses two minima, two maxima and two saddle points on the angular momentum sphere. For the parameters used in the program, the energy at the minima is $E_{\min } / j^{2}=1 /\left(2 I_{z}\right)=0.5$, at the maxima $E_{\max } / j^{2}=1 /\left(2 I_{x}\right)=1.5$, and at the saddle point $E_{\text {sad }} / j^{2}=1 /\left(2 I_{y}\right)=1$. This explains the restriction of the quantum energy eigenvalues to the classically allowed region $E_{\min }<E<E_{\max }$. Furthermore, the quantum state density at the extrema is approximately equal to the period of the classical orbits divided by $2 \pi$, which explains the spike in the figure at the location of saddle point energy because the period at the saddle point is infinite. Note that for $j \rightarrow \infty$ the quantum density also diverges at the saddle point energy. An additional application of the angular momentum operators can be found in section 5 below.

\section{Two-dimensional systems}

For a particle bound in a two-dimensional potential, the Hamiltonian

$$
\hat{H}=\frac{\hat{p}_{1}^{2}}{2 m}+\frac{\hat{p}_{2}^{2}}{2 m}+\hat{V}\left(\hat{x}_{1}, \hat{x}_{2}\right)
$$

can be conveniently expressed in the way described above by means of the tensor product, i.e. the Kronecker product kron provided by MATLAB . Denoting the position operator for one degree of freedom as $\hat{x}$ and the corresponding identity operator as $\hat{I}$, the position operator for the two particles are $\hat{x}_{1}=\hat{x} \otimes \hat{I}$ and $\hat{x}_{2}=\hat{I} \otimes \hat{x}$. The same 
expressions appear for the momentum operators.

As an example the program described below computes the lowest energy eigenvalues for the Pullen-Edmonds potential [5]

$$
V\left(x_{1}, x_{2}\right)=\frac{1}{2} x_{1}^{2}+\frac{1}{2} x_{2}^{2}+\alpha x_{1}^{2} x_{2}^{2}
$$

for $\alpha=0.5$. This potential has been employed in a number of studies related to quantum chaos and also found applications to various molecular systems. The symmetry group of the Hamiltonian is $C_{4 v}$ and the eigenstates can be classified by the irreducible representation $\mathcal{A}_{1,2}, \mathcal{B}_{1,2}$ and $\mathcal{E}[5]$. The wave functions with symmetry $\mathcal{A}_{1}$ or $\mathcal{B}_{2}$ are symmetric if $x_{1}$ and $x_{2}$ are interchanged, those with $\mathcal{A}_{2}$ or $\mathcal{B}_{1}$ symmetry antisymmetric. If, on the other hand, the $x_{j}$ are changed to $-x_{j}$, the $\mathcal{A}_{1}$ or $\mathcal{B}_{1}$ wave functions are unaffected and the $\mathcal{A}_{2}$ or $\mathcal{B}_{2}$ wave functions change sign. In a harmonic oscillator expansion

$$
\varphi\left(x_{1}, x_{2}\right)=\sum_{n, m=0}^{\infty} C_{n m} \varphi_{n}\left(x_{1}\right) \varphi_{m}\left(x_{2}\right)
$$

the coefficients satisfy $C_{n m}=C_{m n}$ for $\mathcal{A}_{1}$ or $\mathcal{B}_{2}$ and $C_{n m}=-C_{m n}$ for $\mathcal{A}_{2}$ or $\mathcal{B}_{1}$. In addition only coefficients with even indices appear for $\mathcal{A}_{1}$ or $\mathcal{B}_{1}$ and only coefficients with odd indices for for $\mathcal{A}_{2}$ or $\mathcal{B}_{2}$. The states with $\mathcal{E}$ symmetry are twofold degenerate. The following program generates the one-dimensional operators as $N \times N$ matrices and constructs the Hamiltonian as $N^{2} \times N^{2}$ matrices using the tensor product as described above. The first $n_{\text {out }}$ eigenvalues are displayed on the screen and the wave function of state number $n_{\text {plot }}$ is finally plotted.

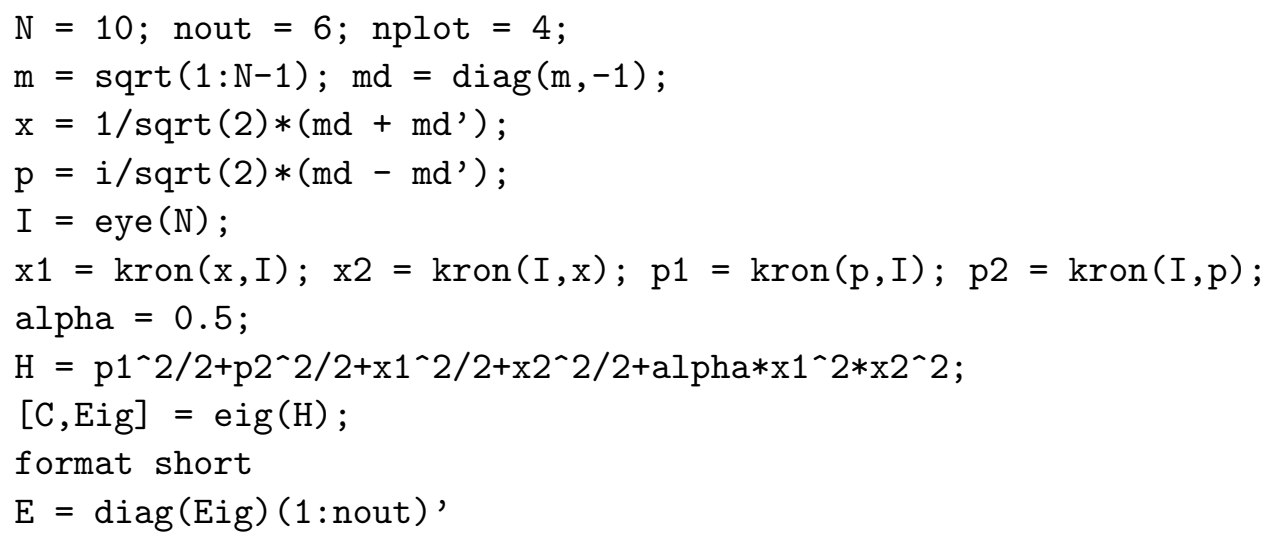

This resulting eigenvalues
1.0980
2.2634
2.2634
$3.2791 \quad 3.5157$
3.7214

agree very well with those given in [6] for the ground state energies with $\mathcal{A}_{1}, \mathcal{E}, \mathcal{B}_{1}$ and $\mathcal{B}_{2}$ symmetry:
1.0980
2.2634
3.2789
3.7223

In order to identify the symmetry of the states calculated numerically, the matrix of the expansion coefficients is displayed by means of

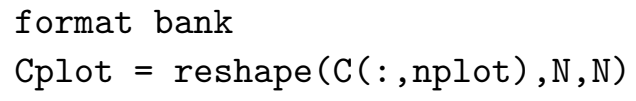


for the state $N$ with energy 3.27914, whose wave function is subsequently plotted. One observes indeed that for this state the matrix is antisymmetric and that all entries with odd indices vanish as required for a symmetry $\mathcal{B}_{1}$. This symmetry is, of course, also visible if the wave function $\varphi\left(x_{1}, x_{2}\right)$ is explicitly calculated and plotted as a color map. The following program lines first compute the one-dimensional harmonic oscillator wave functions $\varphi_{n}(x)$ iteratively which are then stored in the matrix hermval as described in [1]. Finally the expansion (14) is carried out as the scalar product psi $=$ hermval $*$ Cplot $*$ hermval' and plotted:

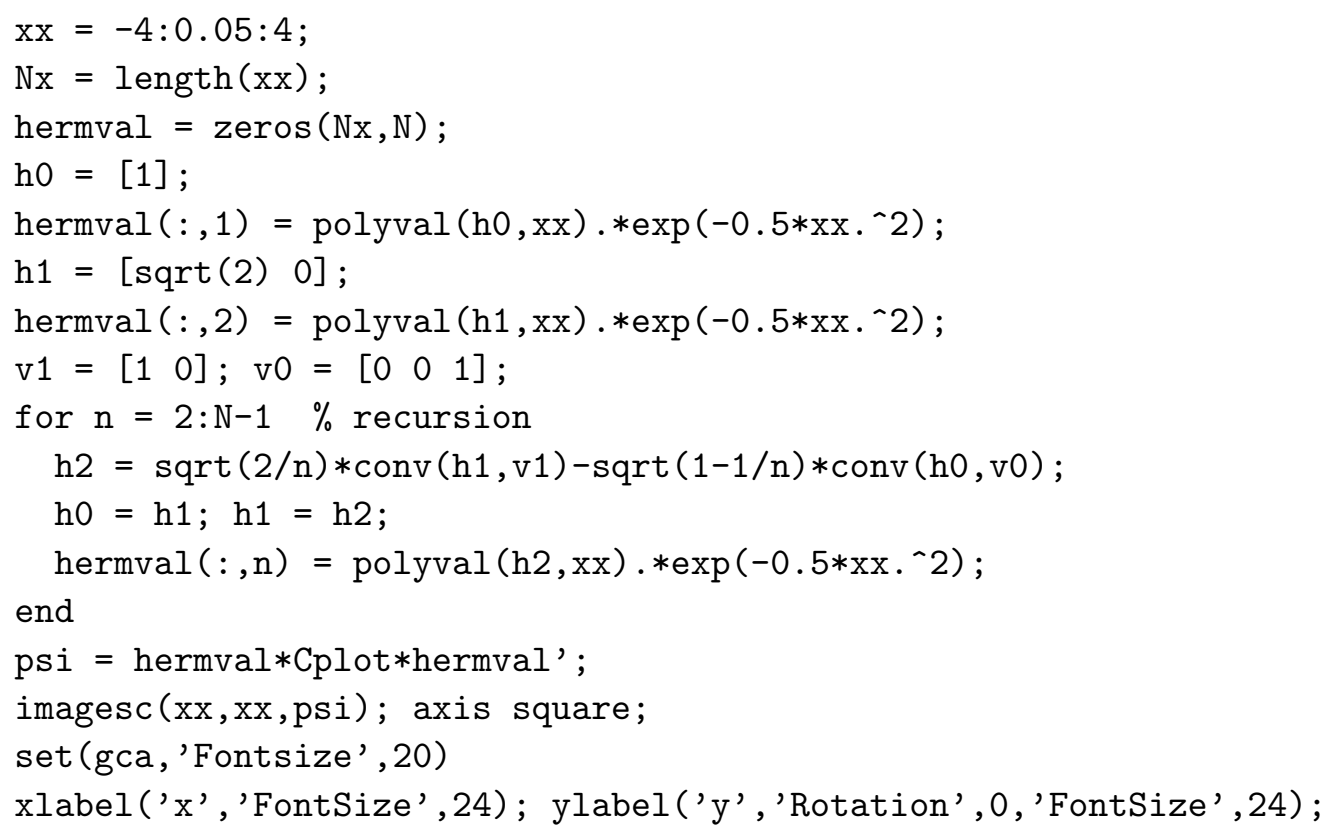

For more pictures of Pullen-Edmonds eigenfunctions see, e.g., [7].

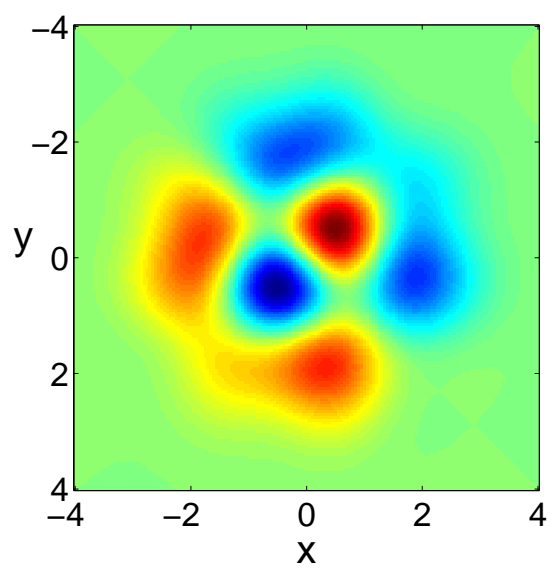

Figure 5. Pullen-Edmonds potential: Color map of the wave function of the lowest eigenstate with symmetry $\mathcal{B}_{1}$. 


\section{Many-particle systems}

A prominent example of a many-particle quantum system is the $N$-particle BoseHubbard system [8], which is one of the basic models studied in theoretical investigations of the dynamics of many particles on a lattice. Numbering the lattice sites by $n$, the operators $\hat{a}_{n}$ and $\hat{a}_{n}^{\dagger}$ with $\left[\hat{a}_{n}, \hat{a}_{n}^{\dagger}\right]=1$ describe annihilation and creation of a particle at site $n$ and $\hat{a}_{n}^{\dagger} \hat{a}_{n}$ is the particle number operator at site $n$. These operators for different

sites commute. Then the hermitian operators $\hat{a}_{n+1}^{\dagger} \hat{a}_{n}$ describe the destruction of a particle at site $n$ and the creation at site $n+1$, and $\hat{a}_{n}^{\dagger} \hat{a}_{n+1}+\hat{a}_{n+1}^{\dagger} \hat{a}_{n}$ the hopping of a particle between these sites. If the particles interact with each other the interaction energy is proportional to the product of the particle number operators. In many cases this interaction is short ranged, so that only the interaction of particles on the same site must be taken into account. Let us confine ourselves here to the simple case of a two-site system, the Bose-Hubbard dimer, with Hamiltonian

$$
\hat{H}=\epsilon\left(\hat{a}_{1}^{\dagger} \hat{a}_{1}-\hat{a}_{2}^{\dagger} \hat{a}_{2}\right)+v\left(\hat{a}_{1}^{\dagger} \hat{a}_{2}+\hat{a}_{2}^{\dagger} \hat{a}_{1}\right)+c\left(\hat{a}_{1}^{\dagger} \hat{a}_{1}-\hat{a}_{2}^{\dagger} \hat{a}_{2}\right)^{2},
$$

where $\pm \epsilon$ are the site energies, $v$ the hopping and $c$ the interaction strength. The Hamiltonian commutes with the particle number operator $\hat{N}=\hat{a}_{1}^{\dagger} \hat{a}_{1}+\hat{a}_{2}^{\dagger} \hat{a}_{2}$, i.e. the particle number $N$ is conserved.

It is of interest to realize that the Bose-Hubbard dimer also appears naturally for a collection of $N$ bosonic atoms in a double-well potential, which is deep enough so that only the lowest state in each well is populated. In this two-mode approximation the system can be described by the Hamiltonian (15).

The following MATLAB program calculates the eigenvalues of the Hamiltonian (15). First the creation and annihilation operators at the two sites are constructed by means of the kron product as well as the Hamiltonian, whose eigenvalues and eigenstates are then calculated for $N=24$, i.e. a matrix dimension of $N_{p}=N+1=25$ for the single particle operators $\hat{a}$ :

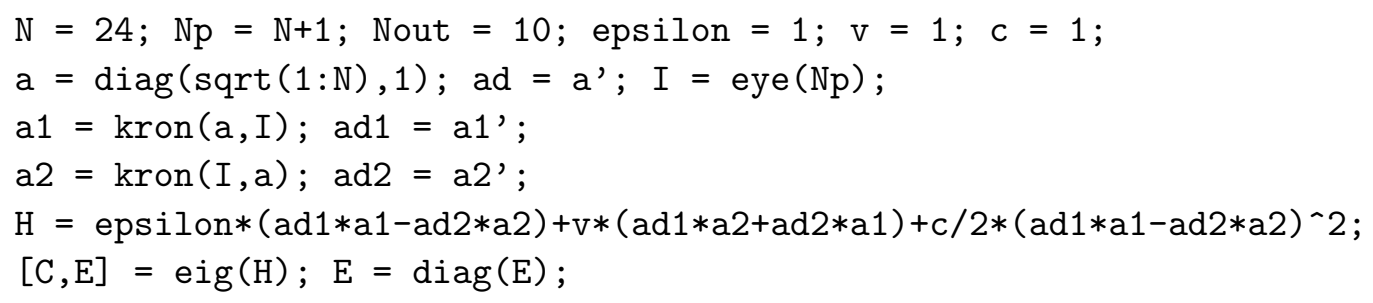

One should be aware of the fact that the many-particle operators are represented by $N_{p}^{2} \times N_{p}^{2}$ matrices and therefore one obtains $N_{p}^{2}=625$ energy eigenvalues, however not all of them are fully converged because of the restricted basis set $\left|n_{1}, n_{2}\right\rangle, n_{1,2}=$ $0,1, \ldots, N$. All eigenstates populating only this restricted basis set are accurately represented, i.e. those with particle numbers up to $N$, a number of $N_{p}\left(N_{p}+1\right) / 2$ states. To illustrate this, the following program lines compute for all calculated eigenstates the expectation values $\langle\hat{N}\rangle$ of the number operator, which agree with the exact particle number for the eigenstates because $\hat{H}$ and $\hat{N}$ commute. These values are subsequently ordered. There are $N^{\prime}+1$ eigenstates for each value of $N^{\prime}=\langle\hat{N}\rangle \leq N$, and therefore a total number of $N_{p}\left(N_{p}+1\right) / 2$, which are converged for the chosen basis size $N_{p}$. Finally the converged energy eigenvalues are plotted as a function of the particle number $N^{\prime}$. 

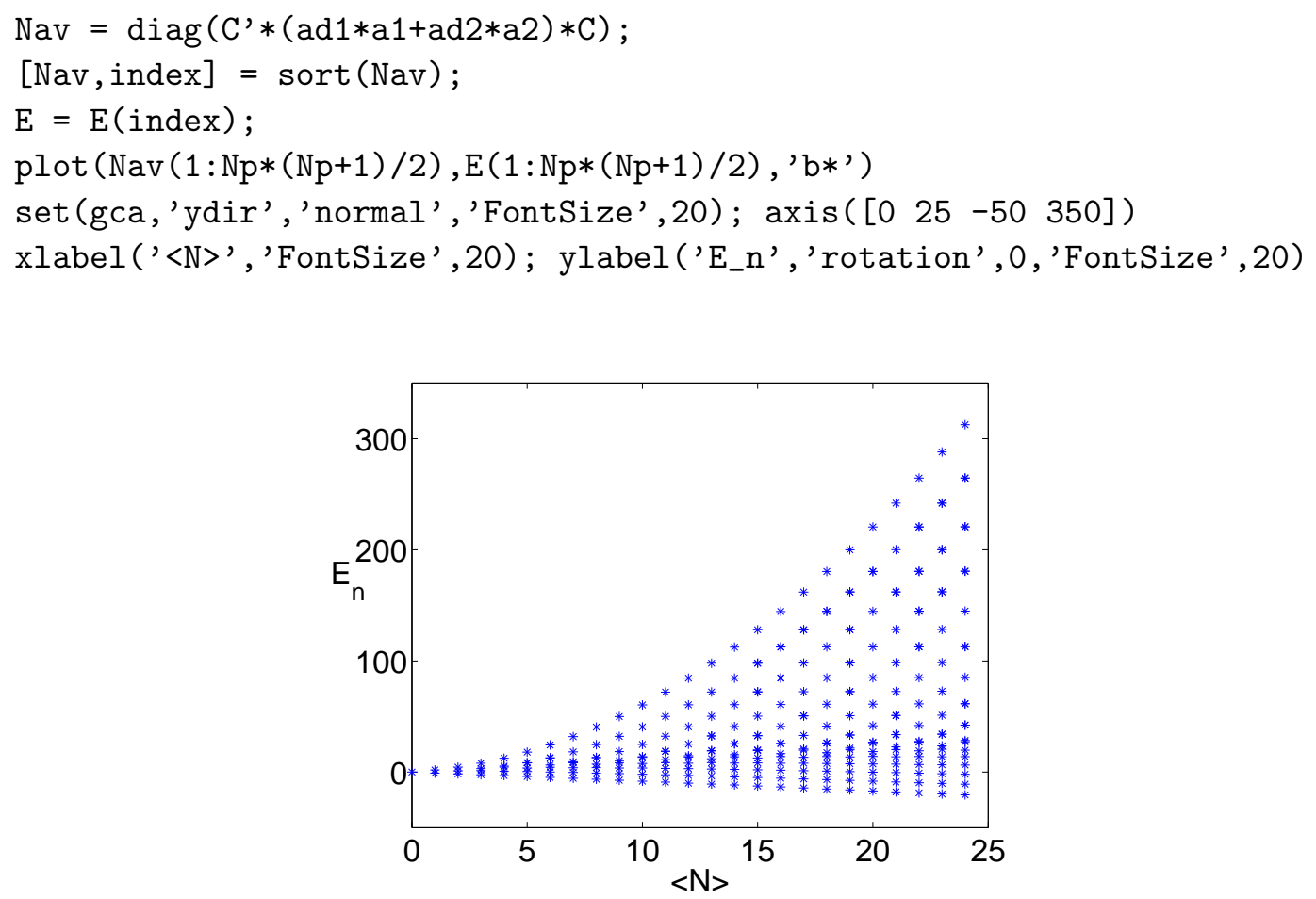

Figure 6. Bose-Hubbard dimer: Energy eigenvalues as a function of the particle number $N$.

In many applications, one is only interested in the eigenvalues and eigenstates of the Hamiltonian for a prescribed value $N$ of the particle number. Then one can make use of the following trick: An extra term $-\lambda\left(\hat{a}_{1}^{\dagger} \hat{a}_{1}+\hat{a}_{2}^{\dagger} \hat{a}_{2}-N\right)(\lambda \gg \epsilon, v)$ is added to the Hamiltonian which does not affect the states with the given particle number $N$ but which shifts the states with different particle numbers to high energies. The desired eigenvalues can then be obtained by extracting the low-energy part of the spectrum of the resulting effective Hamiltonian as implemented in the following program:

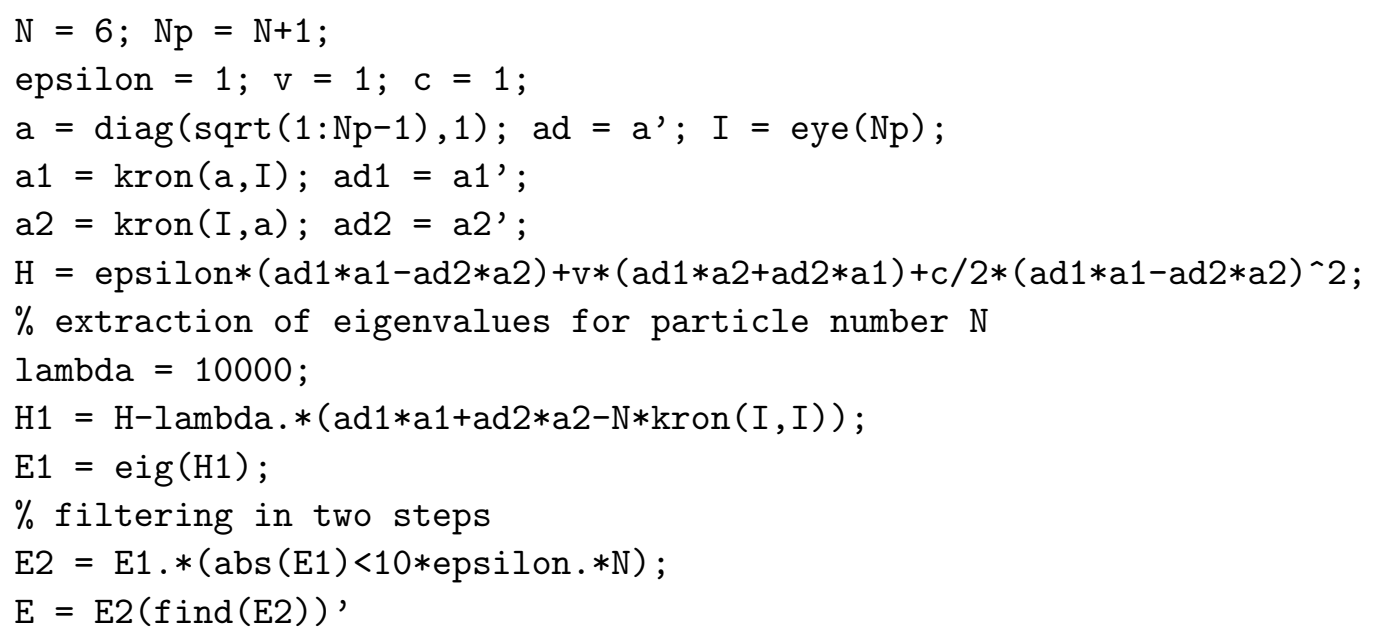

Calculated are the seven eigenvalues for $N=6$ with the result 
A more sophisticated alternative method leading to the same results for the eigenvalues consists in computing the Hamiltonian $\hat{H}_{N}$ for a fixed particle number $N$ by projecting the original Hamiltonian $\hat{H}$ on the corresponding eigenstates of the number operator $\hat{a}_{1}^{\dagger} \hat{a}_{1}+\hat{a}_{2}^{\dagger} \hat{a}_{2}$. Since the latter is a represented by a diagonal matrix, the implementation is rather straightforward:

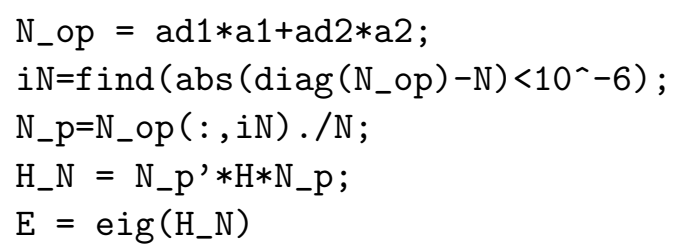

For the Bose-Hubbard dimer, there is, however, a much more convenient way to describe the system for a fixed particle number $N$. The Jordan-Schwinger representation

$$
\begin{aligned}
& \hat{J}_{x}=\left(\hat{a}_{1}^{\dagger} \hat{a}_{2}+\hat{a}_{2}^{\dagger} \hat{a}_{1}\right) / 2, \\
& \hat{J}_{y}=\left(\hat{a}_{1}^{\dagger} \hat{a}_{2}-\hat{a}_{2}^{\dagger} \hat{a}_{1}\right) / 2 \mathrm{i}, \\
& \hat{J}_{z}=\left(\hat{a}_{1}^{\dagger} \hat{a}_{1}-\hat{a}_{2}^{\dagger} \hat{a}_{2}\right) / 2
\end{aligned}
$$

transforms the system to angular momentum operators. The Hamiltonian (15) then takes the form

$$
\hat{H}=2 \epsilon \hat{J}_{z}+2 v \hat{J}_{x}+2 c \hat{J}_{z}^{2}
$$

and the total angular momentum is $\hat{J}=\hat{N} / 2$. Using the matrix representation of angular momentum operators discussed in section 3, this Hamiltonian can be easily coded in the following MATLAB program:

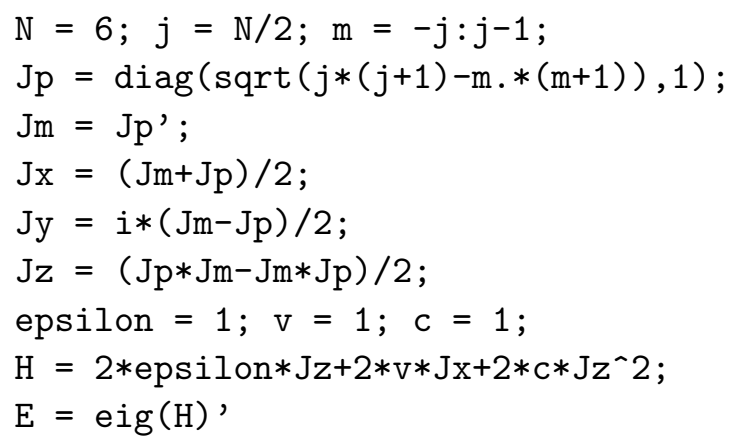

The results agree precisely with those given above for $N=6$.

In the case of high matrix dimensions time and memory can be saved by using sparse matrices. In MATLAB this can be implemented straightforwardly, e.g. the sparse matrix representation of the annihilation operator and the identity in an $N+1$-dimensional space are given by a $=\operatorname{sparse}(\operatorname{diag}(\operatorname{sqrt}(1: \mathrm{N}), 1))$ and $\mathrm{I}=$ speye $(\mathrm{N}+1)$ respectively. This automatically leads to a sparse matrix representation of the Hamiltonian $\hat{H}$. This already becomes relevant if the Bose-Hubbard dimer is extended by an additional site. Such a Bose-Hubbard trimer, as described by the Hamiltonian

$$
\hat{H}=\sum_{j=1}^{3}\left[-\frac{K}{2}\left(\mathrm{e}^{\mathrm{i} \Phi / 3} \hat{a}_{j+1}^{\dagger} \hat{a}_{j}+\mathrm{e}^{-\mathrm{i} \Phi / 3} \hat{a}_{j}^{\dagger} \hat{a}_{j+1}\right)+\frac{U}{2} \hat{a}_{j}^{\dagger} \hat{a}_{j}^{\dagger} \hat{a}_{j} \hat{a}_{j}\right]
$$


was considered in [9]. Here we identify site number 4 with site number 1, i.e. the three sites form a triangular structure which constitutes a minimal model for a superfluid circuit of a Bose-Einstein condensate. The additional phase factors in the hopping term describe the influence of an applied magnetic flux $\Phi$ (in scaled dimensionless units) or alternatively a rotation of the system with a frequency $\Omega \propto \Phi$ (see $[9$ for more details).

The following program computes the eigenvalues $E_{n}$, eigenstates $|n\rangle$ and the expectation values of the current $J_{n}=\langle n|\hat{J}| n\rangle=\left\langle n\left|\frac{\partial \hat{H}}{\partial \Phi}\right| n\right\rangle$ and plots the results:

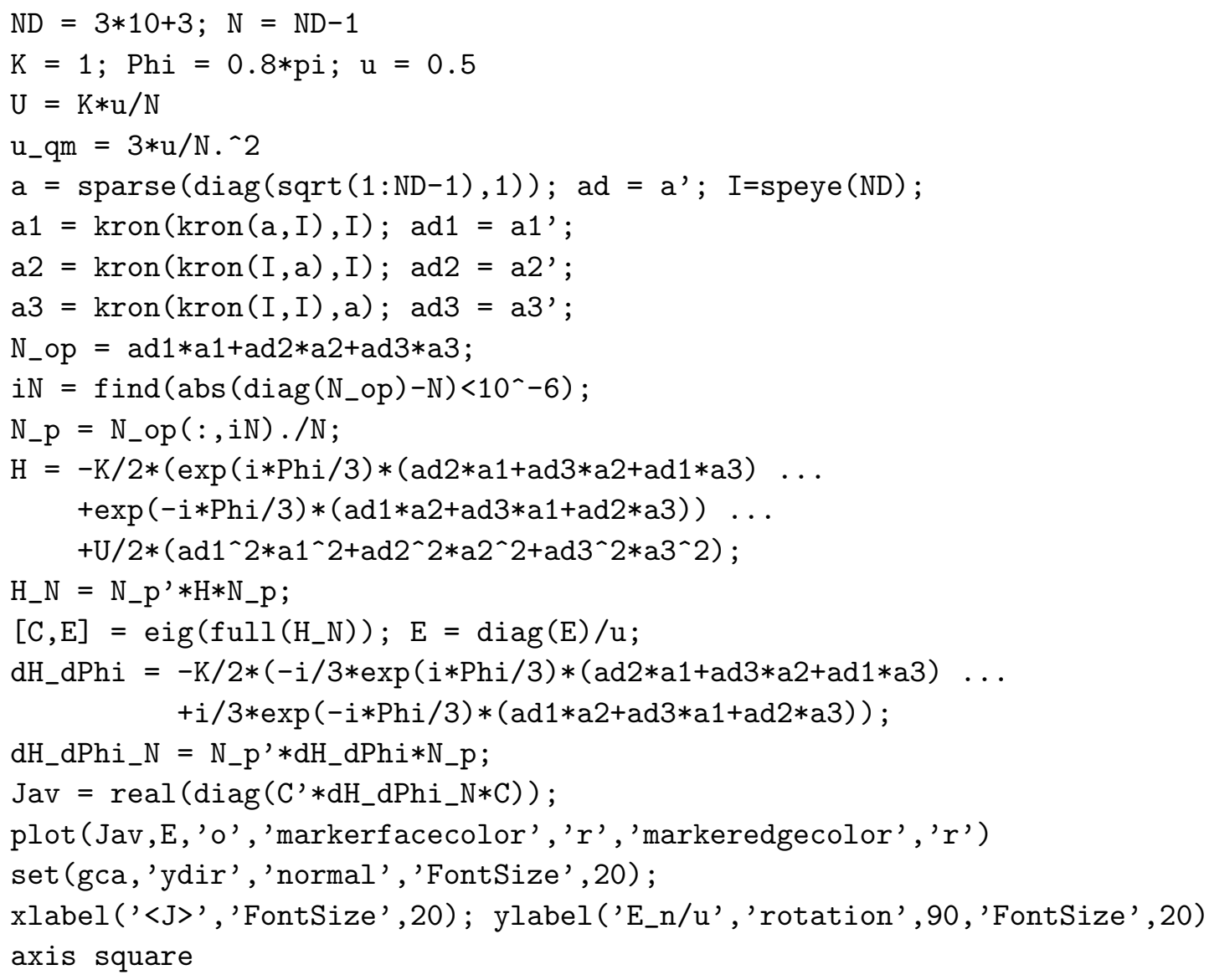

(depending on the MATLAB version used it may be required to replace eig(H_N) by $\operatorname{eig}\left(\mathrm{full}\left(\mathrm{H} \_\mathrm{N}\right)\right)$.)

Figure 7 shows the distribution of the eigenstates with respect to the eigenenergies and the currents. For noninteracting particles, $u=U N / K=0$, the current $\hat{J}$ commutes with the Hamiltonian and each eigenstate carries a quantized current, forming a triangle in the energy-current plane as explained in [9]. For an integer filling $N=30=10 \cdot 3$ of the trimer, the eigenstates are more and more redistributed along the line indicating zero current with increasing interaction $u$. In the high interaction limit, even the ground state becomes a zero current and thus an insulator state. This is a signature of a socalled superfluid to Mott insulator transition, illustrated by the first four panels. In contrast, for a non-integer filling per site the ground state always carries a current even in the strong interaction limit as shown for $N=31$ and $N=32$ in the last two panels. More about the rich behavior of this three site Bose-Hubbard system can be found in 9 . 

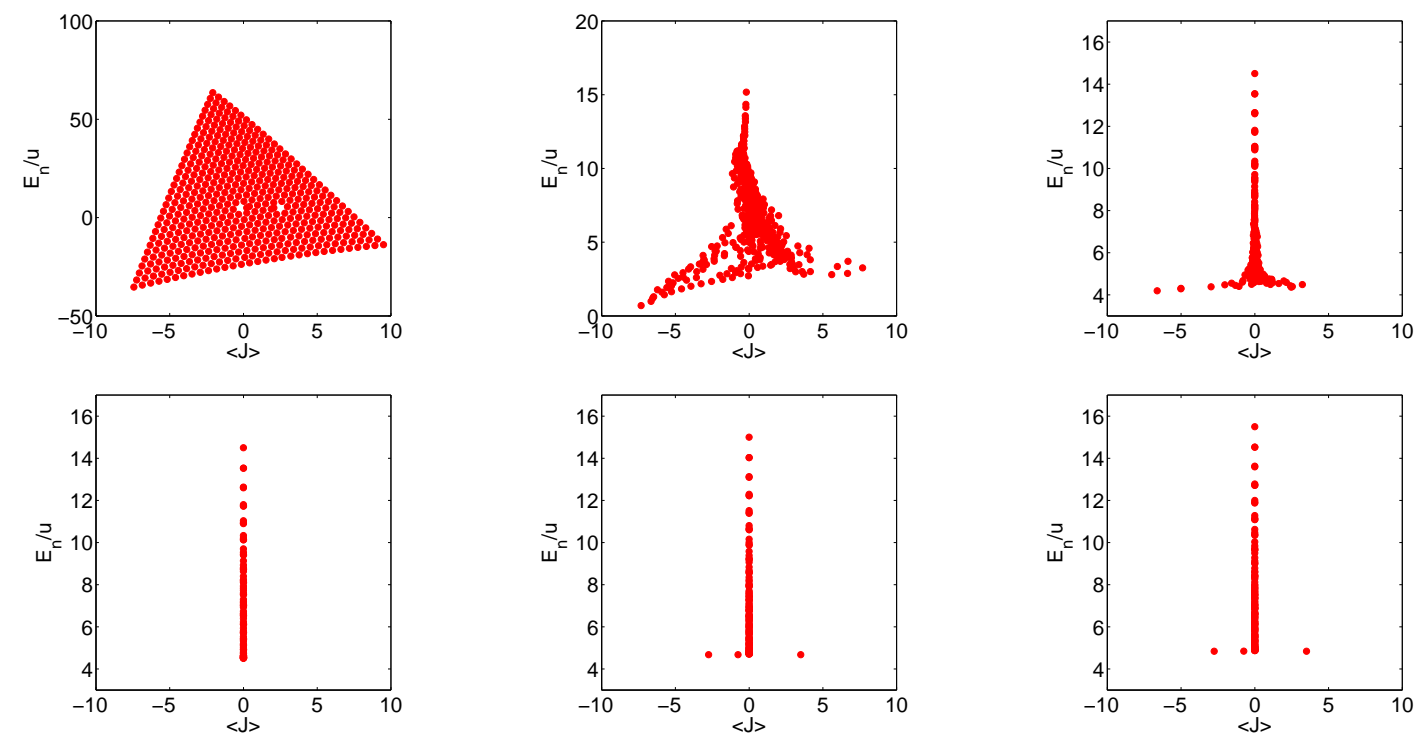

Figure 7. Trimer: Current $\langle J\rangle$ and eigenenergies $E_{n} / u$ of the Bose-Hubbard trimer for $K=1, \Phi=0.8 \pi$. First four panels: $N=30$ particles and interaction strength $u=U N / K=0.5,5,50,50000$ respectively. Last two panels: $N=31, N=32$ and $u=50000$.

\section{The Lindblad master equation for an open quantum system}

Open quantum systems are relevant in various fields including electronic transport in semiconductors and nanostructures [10] and cavity QED [11. Another example of an open system is given by Bose-Einstein condensates in optical lattices which are subject to a decay process due to coupling to an environment, a setup studied recently experimentally (see e.g. [12]) and theoretically using different approaches [13 18]. For weak decay such systems can be described by means of a Born-Markov approximation leading to a Lindblad master equation for the system's density matrix [19]. For the Bose-Hubbard dimer of section 5 the Lindblad master equation can be written as (see e. g. [17])

$$
\dot{\hat{\rho}}=-\mathrm{i}[\hat{H}, \hat{\rho}]-\frac{\gamma}{2}\left(\hat{a}_{2}^{\dagger} \hat{a}_{2} \hat{\rho}+\hat{\rho} \hat{a}_{2}^{\dagger} \hat{a}_{2}-2 \hat{a}_{2} \hat{\rho} \hat{a}_{2}^{\dagger}\right)
$$

where the second term models a particle decay from site 2 with rate $\gamma$ and the first term corresponds to the hermitian part of the time evolution with the two site BoseHubbard Hamiltonian $\hat{H}$ as given in equation (15). In [18] it was shown that this equation accurately describes tunneling decay from the dimer into a weakly coupled optical lattice.

In the following we consider the situation where initially $N$ particles are in the second well of the Bose-Hubbard dimer whereas the first well is empty. The corresponding initial wavefunction and density matrix at time $t=0$ are then given by $\left|\psi_{0}\right\rangle=|0\rangle \otimes|N\rangle$ and $\hat{\rho}(0)=\left|\psi_{0}\right\rangle\left\langle\psi_{0}\right|$ respectively. Using the matrix representations introduced in the previous sections the following MATLAB code propagates the initial 
density matrix according to equation $(19)$ by means of a predictor corrector integrator [20]. The expectation values of the time-dependent site occupations are then obtained via $n_{j}(t)=\operatorname{trace}\left(\hat{\rho}(t) \hat{a}_{j}^{\dagger} \hat{a}_{j}\right)$.

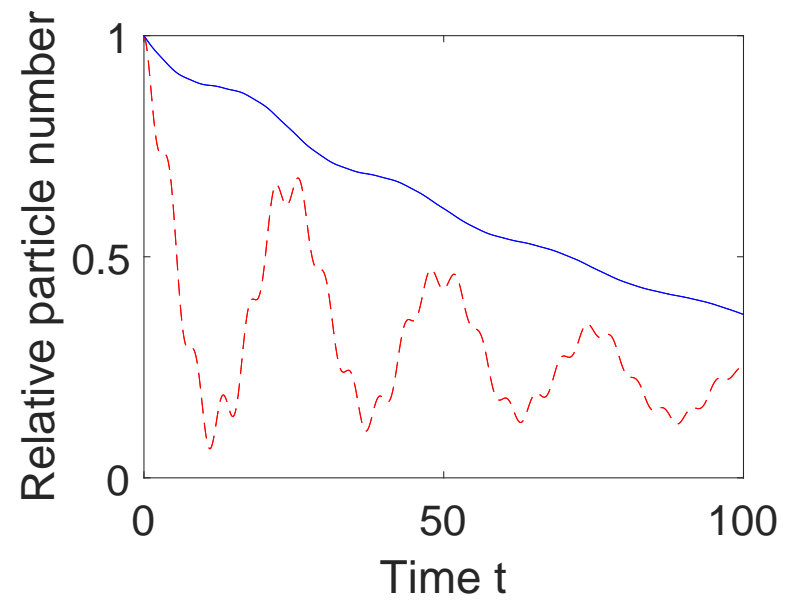

Figure 8. Relative total particle number $\left(n_{1}(t)+n_{2}(t)\right) / N$ (solid line) and relative particle number in the second well $n_{2}(t) / N$ (dashed line) as a function of time for an open Bose-Hubbard dimer with $\epsilon=0, v=0.3, c=0.6, N=2$ and decay with the rate $\gamma=0.02$ from the second well. Scaled units with $\hbar=1$ are used.

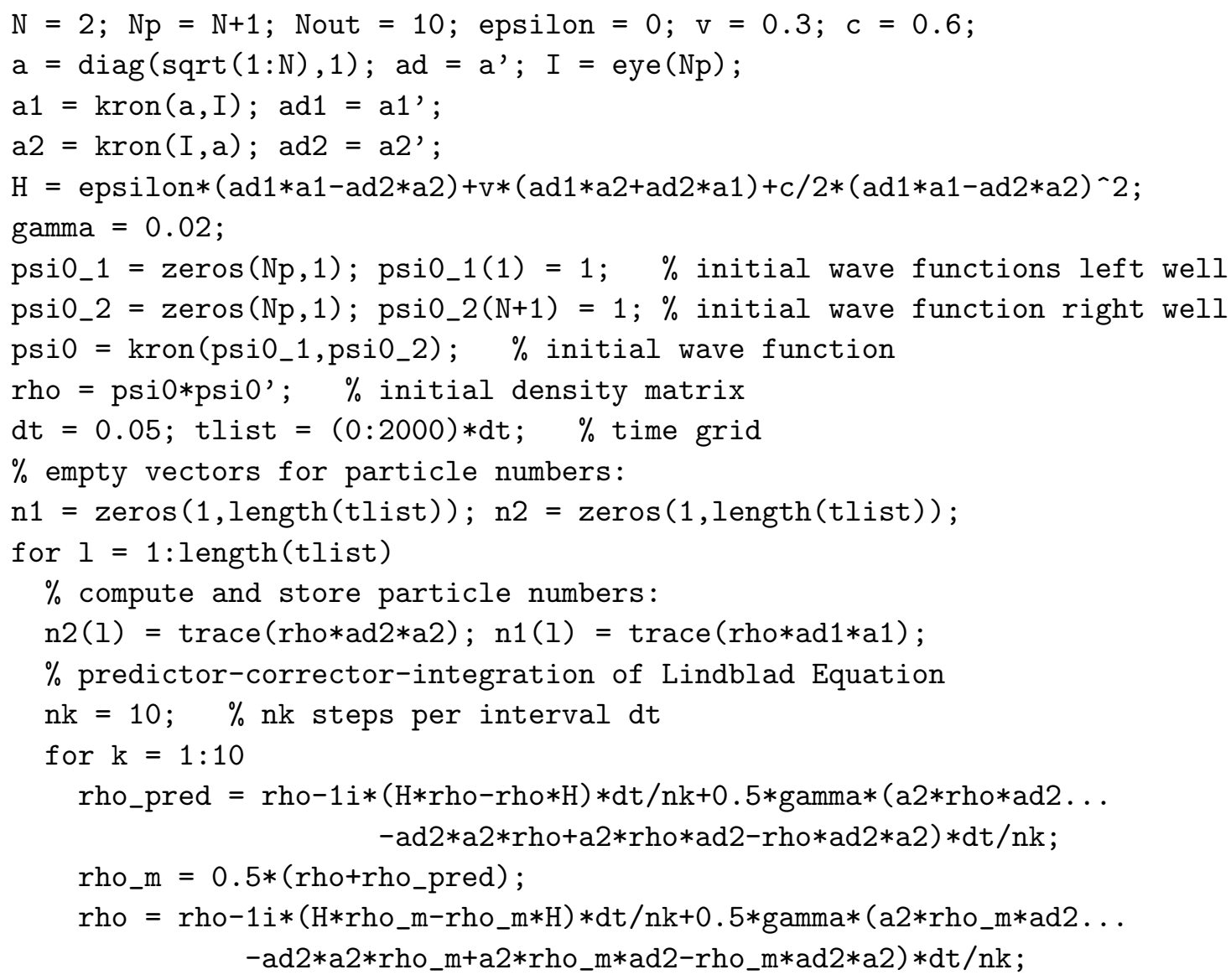




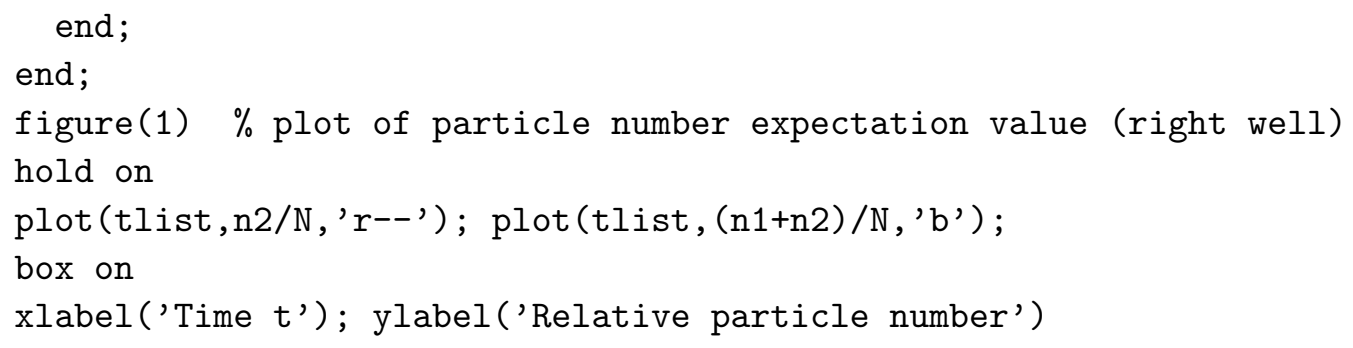

Figure 8 shows the resulting decay dynamics of the relative total particle number $\left(n_{1}(t)+n_{2}(t)\right) / N$ (solid line) and the relative particle number in the second well $n_{2}(t) / N$ for a symmetric double well with $\epsilon=0$, tunneling coefficient $v=0.3$, interaction constant $c=0.6$, decay rate $\gamma=0.02$ and an initial particle number $N=2$.

In a non-interacting open dimer, the occupation $n_{2}(t) / N$ would yield exponentially damped cosine-shaped Rabi oscillations. We clearly observe how this behavior is modified by the interaction between the particles. Various Fourier components occur which result from different excitation energies in the spectrum of the interacting bosonic system.

\section{Concluding remarks}

Matrix representation techniques were presented and illustrated by a variety of different examples demonstrating both their simplicity and wide applicability. These qualities make them suitable for use in research projects as well as quantum mechanics courses for undergraduate and graduate students.

\section{Acknowledgments}

The authors would like to thank Eva-Maria Graefe for careful reading of the manuscript and for all valuable comments and suggestions.

\section{References}

[1] M. Glück and H. J. Korsch, Eur. J. Phys. 23 (2002) 413

[2] T. Hartmann, F. Keck, H. J. Korsch, and S. Mossmann, New J. Phys. 6 (2004) 2

[3] L. D. Landau and E. M. Lifshitz, Quantum Mechanics, Pergamon Press, New York, 1977

[4] L. E. Ballentine, Quantum Mechanics - A Modern Development, World Scientific, Singapore, 2006

[5] R. A. Pullen and A. R. Edmonds, J. Phys. A 14 (1981) L477

[6] P. Amore and F. M. Fernandez, Phys. Scripta 80 (2009) 055002

[7] S. K. Joseph,, 2014. www.youtube.com/watch?v=gVAyZ47Iw7Q

[8] L. Pitaevskii and S. Stringari, Bose-Einstein Condensation, Oxford University Press, Oxford, 2003

[9] G. Arwas, A. Vardi, and D. Cohen, Phys. Rev. A 89 (2014) 013601

[10] M. Di Ventra, Electrical Transport in Nanoscale Systems, Cambridge University Press, Cambridge, 2008

[11] M. O. Scully and M. S. Zubairy, Quantum Optics, Cambridge University Press, Cambridge, 1997

[12] P. Würtz, T. Langen, T. Gericke, A. Koglbauer, and H. Ott, Phys. Rev. Lett. 103 (2009) 80404 
[13] K. Rapedius, C. Elsen, D. Witthaut, S. Wimberger, and H. J. Korsch, Phys. Rev. A 82 (2010) 063601

[14] A. U. J. Lode, A. I. Streltsov, O. E. Alon, H.-D. Meyer, and L. S. Cederbaum, J. Phys. B 42 (2009) 044018

[15] K. Rapedius and H. J. Korsch, Phys. Rev. A 86 (2012) 025601

[16] E. M. Graefe, H. J. Korsch, and A. E. Niederle, Phys. Rev. Lett 101 (2008) 150408

[17] D. Witthaut, F. Trimborn, and S. Wimberger, Phys. Rev. Lett. 101 (2008) 200402

[18] K. Rapedius, J. Phys. B 46 (2013) 125301

[19] H.-P. Breuer and F. Petruccione, The Theory of Open Quantum Systems, Oxford University Press, Oxford, 2002

[20] W. H. Press, S. A. Teukolsky, W. T. Vetterling, and B. P. Flannery, Numerical Recipes, Cambridge University Press, London, 3. edition, 2007 